\title{
A narrative review of a type of pancreatitis worthy of attention: acute pancreatitis associated with pancreatic tumors-current problems and future thinking
}

\author{
Anjiang Gou", Zhe Liu", Zhihuan Xiao, Guichen Li, Yuanhong Xu, Shaowei Song, Kejian Guo, Gang Ma \\ Department of Pancreatic-Biliary Surgery, First Hospital of China Medical University, Shenyang, China \\ Contributions: (I) Conception and design: A Gou, Z Liu; (II) Administrative support: G Ma, G Li, Y Xu, S Song, K Guo; (III) Provision of study \\ materials or patients: A Gou, Z Liu; (IV) Collection and assembly of data: A Gou, Z Liu, Z Xiao; (V) Data analysis and interpretation: A Gou, Z Liu, \\ Z Xiao; (VI) Manuscript writing: All authors; (VII) Final approval of manuscript: All authors. \\ \#These authors contributed equally to this work. \\ Correspondence to: Dr. Gang Ma. Department of Pancreatic-Biliary Surgery, First Hospital of China Medical University, 155 North Nanjing St., \\ Shenyang 110001, China. Email: magangzy@163.com.
}

\begin{abstract}
Objective: Our purpose is to explain the onset, diagnosis, and treatment of pancreatic tumor-associated pancreatitis (PTP), and inform clinicians about the management of PTP. It is hoped that clinicians can gain some experience and inspiration from this review, so that patients can obtain better treatment results.

Background Acute pancreatitis (AP) is an inflammatory disease, and pancreatic tumors are one of the causes of pancreatitis. When pancreatic tumors and pancreatitis exist at the same time, and there is a "connection" between them, this type of pancreatitis is referred to as PTP. The manifestations of PTP can be as follows: (I) AP is the first symptom of pancreatic tumors; (II) pancreatitis is found in patients after pancreatic tumor diagnosis or during pancreatic tumor surgery. Because pancreatic tumors are not one of the most common causes of pancreatitis, PTP has not attracted the attention of researchers and clinicians, and there is no consistent and clear understanding of the diagnosis and treatment of PTP.

Methods: From the online database PubMed (https://pubmed.ncbi.nlm.nih.gov/) and Web of Science (https://webofknowledge.com/), we use specific retrieval strategies to retrieve relevant articles, and we review and discuss them.

Conclusions: What we need to realize is that PTP is different from ordinary AP. It has its own characteristics in terms of diagnosis and treatment, which requires the attention of clinicians. More importantly, future research should design the best diagnosis and treatment algorithms for PTP.
\end{abstract}

Keywords: Pancreatic tumor-associated pancreatitis (PTP); acute pancreatitis (AP); diagnostic; surgical treatment; classification

Submitted May 20, 2021. Accepted for publication Jul 15, 2021.

doi: $10.21037 /$ gs-21-400

View this article at: https://dx.doi.org/10.21037/gs-21-400

\section{Introduction}

Acute pancreatitis (AP) is an inflammatory reaction of pancreatic tissue self-digestion, edema, and hemorrhage, with or without other organ failure, and is a common clinical acute abdomen. AP is the most common gastrointestinal disease requiring hospitalization, with an annual incidence of 34/100,000 in developed countries (1).
We have newly defined a type of pancreatitis based on clinical practice experience, namely pancreatic tumorassociated pancreatitis (PTP). PTP has many new features in terms of diagnosis and treatment, and it is therefore necessary to describe it in detail.

$\mathrm{AP}$ has various causes and a complex pathogenesis, and its occurrence and development are affected by many factors. 
Most AP is biliary pancreatitis, but in some cases, the cause is difficult to identify. Pancreatitis due to pancreatic tumors is not common, but the existence of this type of pancreatitis should not be ignored, along with the possibility that AP is caused by pancreatic tumors, especially in older patients (2). Pancreatic tumors, such as intraductal papillary mucinous neoplasm (IPMN) and pancreatic cancer, among others, may cause AP. In some published articles, most of them simply discussed pancreatic tumors or pancreatitis, or just compared the epidemiology and risk factors of pancreatic tumors and pancreatitis. Few articles specifically discussed the relationship between pancreatitis and pancreatic tumors. Therefore, based on the relationship between pancreatic tumors and pancreatitis, we put forward our novel point of view. When pancreatic tumors and pancreatitis exist at the same time, and there is a "connection" between them, and this type of pancreatitis is referred to as PTP. At this time, the mechanisms and causal link between pancreatitis and pancreatic tumors are complicated. The manifestations of PTP can be as follows: (I) AP is the first symptom of pancreatic tumors; (II) pancreatitis is found in patients after pancreatic tumor diagnosis or during pancreatic tumor surgery. In this review, we mainly introduce the incidence, diagnosis, and treatment of PTP, and discuss the relationship between pancreatitis and pancreatic tumors, including IPMN, pancreatic cancer, solid pseudopapillary neoplasm (SPEN), and pancreatic cystic tumors, among others. We also discuss future thinking regarding PTP. We present the following article in accordance with the Narrative Review reporting checklist (available at https:// dx.doi.org/10.21037/gs-21-400).

\section{Methods}

The research strategy involved the online databases Web of Science (https://webofknowledge.com/) and PubMed (https://pubmed.ncbi.nlm.nih.gov/). The search strategy included the following key terms: acute pancreatitis, pancreatic, pancreatic tumor, IPMN, pancreatic cancer, pancreatic ductal adenocarcinoma, islet cell tumor, serous cystic neoplasm, mucinous cystic neoplasm, neuroendocrine tumors, solid pseudopapillary neoplasm, pancreatic cystic tumors, diagnosis. Specifically, the research was restricted using variable combinations of the keywords. Articles in English published between January 1970 and April 2021 were searched. Some articles were excluded as they were not related to the topic of discussion.

\section{Incidence}

Despite the rapid growth of medical knowledge and the rapid development of treatment technologies, we still know very little about pancreatic diseases. AP is the most common disease of the pancreas worldwide (1). It is an unpredictable and potentially fatal disease that is difficult to manage. The annual global incidence of AP is 34 per 100,000 people, and it has been increasing worldwide (3). In most high-income countries, gallstones (approximately 45\%) and alcohol (approximately 20\%) are the two main causes of AP (4-7). Followed by idiopathic pancreatitis, other uncommon causes are viral infections, malignant tumors, endoscopic retrograde cholangiopancreatography (ERCP), genetics, autoimmune diseases, hypertriglyceridemia, hypercalcemia, surgical trauma, cystic fibrosis, and complications from drugs or chemotherapy $(4,5,8)$. These causes lead to organelle dysfunction and pathological cellular pathways, and eventually acinar cell death and local and systemic inflammation. It is worth noting that the role of dietary factors in the etiology of pancreatitis is not yet clear, which is an important area of future research (9). But in clinical work it is usually found that people who overeating are more likely to develop pancreatitis than people who eat a normal diet. More research is needed on this.

The cellular events important to the pathogenesis of AP include pathological calcium signaling (10-12), premature trypsinogen activation within macrophages and acinar cells (13-15), mitochondrial dysfunction (16,17), endoplasmic reticulum (ER) stress $(18,19)$, impaired autophagy, and impaired unfolded protein response (UPR) (17). These events are caused by usual acinar cell toxins, such as nicotine, alcohol, and bile acids. Ductal cell dysfunction and intraductal events, such as increased pressure caused by luminal acidification, ductal obstruction, and ductal cell exposure to bile acid, can also indirectly cause these events. The crosstalk between the immune system and acinar cells perpetuates an inflammatory response $(20,21)$.

The possible causes of pancreatitis from pancreatic tumors are as follows: (I) obstruction of the pancreatic duct leads to obstruction of pancreatic juice drainage; (II) local pancreatic necrosis caused by tumor growth; (III) hyperlipidemia, gallbladder stones and drinking; (IV) as there may be the main cause of pancreatitis at the same time, and PTP is rare, pancreatic tumors are ignored, and the timing of surgery is often delayed on account of the 
Table 1 PTP cases in the First Affiliated Hospital of China Medical University from 2010 to 2020

\begin{tabular}{|c|c|c|c|c|c|c|c|}
\hline Salient comparison variable/ histology & PDAC & IPMN & SCN & $\mathrm{MCN}$ & SPEN & ICT & NETs \\
\hline Head & 17 & 22 & 7 & 5 & 0 & 1 & 0 \\
\hline Body and tail & 13 & 12 & 3 & 2 & 2 & 3 & 3 \\
\hline \multicolumn{8}{|l|}{ Severity } \\
\hline Severe & 7 & 5 & 1 & 0 & 0 & 0 & 0 \\
\hline \multicolumn{8}{|l|}{ Operative details } \\
\hline DPS & 0 & 16 & 5 & 3 & 1 & 3 & 2 \\
\hline PD & 29 & 13 & 3 & 4 & 0 & 0 & 0 \\
\hline Total pancreatectomy & 1 & 2 & 1 & 0 & 0 & 1 & 0 \\
\hline Inoperable \pm palliative procedure & 0 & 0 & 0 & 0 & 0 & 0 & 1 \\
\hline
\end{tabular}

PTP, pancreatic tumor-associated pancreatitis; PDAC, pancreatic ductal adenocarcinoma; IPMN, intraductal papillary mucinous neoplasm; SCN, serous cystic neoplasm; MCN, mucinous cystic neoplasm; SPEN, solid pseudopapillary neoplasm; ICT, islet cell tumor; NET, neuroendocrine tumor; DPS, distal pancreatosplenectomy; PD, pancreatoduodenectomy; DP, distal pancreatectomy.

diagnosis of AP. It is worth noting that the pancreatic tumors we cite here include benign and malignant pancreatic tumors, such as pancreatic ductal adenocarcinoma (PDAC), IPMN, islet cell tumor (ICT), pancreatic neuroendocrine tumor (NET), pancreatic cystadenoma, and SPEN, among others. Among them, the most common tumors are PDACs and IPMNs. The statistics of PTP inpatients from the First Affiliated Hospital of China Medical University over the past 10 years is as follows (Table 1): PDACs and IPMNs are the main causes of PTP, and PTP is mostly mild and moderate.

\section{Diagnosis and treatment}

For the diagnosis of AP, there are already accepted standards, which can be regarded as the gold standard, that is at least two of the three following criteria need to be met: (I) typical abdominal pain; (II) serum amylase and/ or lipase elevation at least three times the upper limit of the normal value; (III) findings in accordance with AP on imaging [ultrasound, contrast-enhanced CT (CECT), or MRI] (22). Due to different laboratory techniques for measuring these enzymes, there is no standardized reference range for serum amylase or lipase levels. The upper limit of the normal range for amylase is between 100 and $300 \mathrm{U} / \mathrm{L}$, and 50 and $160 \mathrm{U} / \mathrm{L}$ for lipase (23). Amylase is elevated in diseases such as peptic ulcer, gastrointestinal perforation, infarction, obstruction, abdominal aortic aneurysm, and peritonitis. At the same time, patients with alcoholic or hypertriglyceridemia pancreatitis have normal amylase levels, and similarly, lipase is also elevated in acute cholecystitis, intestinal pathologies, biliary obstruction, and peptic ulcer disease $(24,25)$. Therefore, the limitations of serum amylase and lipase as diagnostic methods for AP are worthy of attention, and diagnosis might be challenging in these populations. Thus, imaging tests can aid in the diagnosis of AP when the diagnosis is in doubt. All these diagnostic criteria are subscribed to by all published guidelines regarding AP (25-28). Strict compliance with the diagnostic criteria for pancreatitis will reduce the misdiagnosis of pancreatitis.

Whilst it is easier to make a diagnosis of AP, it may be more difficult to make a diagnosis of PTP. When considering the etiology of $\mathrm{AP}$ and making a diagnosis of PTP, the suggestive clinical data are as follows: preexisting cyst with recurrent idiopathic episodes; dilated pancreatic duct in a patient without prior history of chronic pancreatitis; diabetes diagnosis; weight loss; mass with 
duct dilatation. In addition, imaging examinations and tumor markers should be combined. Spiral CT, especially multi-slice spiral CT, is a reliable non-invasive screening test that can detect pancreatic cancer with pancreatitis in most patients (29). However, the following situations may occur, resulting in an inability to make a diagnosis of PTP and make the best treatment decision: (I) clinicians are only satisfied with the diagnosis and treatment of patients with pancreatitis, but overlook all sorts of warning signals about pancreatic tumors; (II) some patients have a large amount of exudation and fluid accumulation due to pancreatitis, and ultimately cannot show the characteristics of pancreatic tumors on imaging, and eventually pancreatic tumors cannot be found. Furthermore, some inflammatory pancreatic imaging may mimic PDAC imaging when abnormal, resulting in misdiagnosis; (III) PTP is rare, and its rarity makes it difficult for clinicians to consider it. Usually, the diagnosis of PTP should be considered after all non-tumor factors that cause pancreatitis have been excluded.

According to the 2012 Atlanta revised classification and definition, the AP classification clarifies the two phases of the disease: early and late. Severity is divided into mild, moderate, and severe (22). Mild AP has no organ failure or local and systemic complications, and patients are usually discharged within 1 week. Moderately severe AP is characterized by transient organ failure, or local or systemic complications without persistent organ failure. Severe AP is defined by persistent organ failure (time of duration $>48 \mathrm{~h}$ ). However, the definition of the severity of PTP has not been described in the literature. Whether to use the 2012 Atlanta classification of AP, or whether the impact of different pancreatic tumors and tumor stages will affect the severity of PTP are questions worthy of consideration and research.

Early management includes fluid resuscitation, identification of etiology, nutritional support, and analgesia. The goals of AP management in the first week are to assess severity and assign an appropriate level of care, to ascertain etiology and address a reversible cause, and to optimize nutrition and arrange appropriate follow-up for the majority of patients being discharged. Systemic inflammatory response syndrome (SIRS), which includes body temperature, white blood cell count, heart rate, and respiratory rate, is a commonly used and effective predictor of AP severity and mortality (30). Severe AP is mainly caused by the occurrence of SIRS, and inhibiting inflammation is beneficial to PTP. Up to $80 \%$ of patients with AP will recover and be discharged within a week.
However, beyond week 1, 20\% will deteriorate and will need more long-term care (23). The treatment of PTP not only needs to consider the treatment of pancreatitis, but also needs to consider the treatment of pancreatic tumors. Pancreatic tumors require surgical treatment, and due to the existence of pancreatitis, the operation of PTP is more difficult than for simple pancreatic tumors. Therefore, for patients with benign or borderline pancreatic tumors combined with pancreatitis, if the pancreatic inflammation is severe and the preoperative evaluation is difficult, the pancreatitis should be treated under close observation, and surgical treatment will be considered after the pancreatic inflammation is clearly controlled. If the tumor has a tendency to become malignant during the observation process, multidisciplinary team (MDT) consultation is required to comprehensively consider the difficulty of the operation and the basic state of the patient to make the best treatment decision. For patients with pancreatic cancer and AP, surgery should be performed as soon as possible after the acute phase of pancreatitis is controlled. If the preoperative assessment is that the pancreas is exuding, especially around the pancreatic head, and the duodenal edema is particularly severe, the risk of surgery can be reassessed as soon as possible (recommended within half a month to 1 month) after active treatment to determine the time and method of surgery. Surgical methods can be divided into: pancreaticoduodenectomy, pancreatic body and tail resection, and spleen-preserving pancreatic body and tail resection, among others. However, as such patients have pancreatitis, and most of the tumors are closely related to the pancreatic duct, local excision of pancreatic tumors is generally not recommended. At the same time, due to the presence of pancreatic inflammation, it is difficult to perform splenic vessel-preserving pancreatic body and tail resection (Kimura procedure). Such patients are advised to choose this procedure carefully.

\section{Discussion}

The postoperative survival rate of pancreatic tumors is very low, and pancreatitis is a factor that affects the difficulty of pancreatic surgery and postoperative survival. Among the pancreatic tumors, PDAC is often associated with pancreatitis $(31,32)$. However, compared with pancreatic cancer, there are more patients with AP in IPMN patients (29). The possible reason is that compared with the obstruction caused by solid tumors, the obstruction of the main or branch ducts caused by abundant mucus 
secretion is more likely to cause AP and/or pain (33). In a study of IPMN patients, AP was the presenting symptom in approximately $35 \%$ of patients. In $85 \%$ of IPMN patients, $\mathrm{AP}$ presents as a mild edematous-interstitial inflammation that is self-limiting, and severe AP is rare in patients with IPMN (34). Several studies have shown that AP in IPMN patients is usually mild and recurrent (34-36). Research also shows that there does not seem to be a difference in the incidence of AP between benign and malignant IPMN (34). For AP patients with pancreatic duct dilatation in the absence of a prior history of pancreatitis or chronic pancreatitis, main-duct IPMN should be considered as an etiology, and main-duct IPMNs have high malignant potential, so it is crucial to recognize and treat them accordingly (23). AP is relatively rare in serous cystic neoplasm (SCN) and mucinous cystic neoplasm (MCN), and the possible cause is compression of the pancreatic duct caused by cystic lesions $(37,38)$. SPENs of the pancreas cause AP more rarely. There are only a few cases reported in the literature, and the possible cause of AP is that the mass compresses the main pancreatic duct $(39,40)$.

Pancreatic cancer is another important cause, accounting for approximately $1 \%$ of all AP causes (41). According to a study report, AP occurred in about $3 \%$ of pancreatic cancer patients, and approximately $10 \%$ of patients with pancreatic cancer found pathological stigmata of AP, such as fat necrosis and inflammation, in surgical and/or pathological specimens (42). In patients over the age of 40 years, a follow-up imaging study or endoscopic ultrasonography (EUS) is crucial to rule out a tumor. AP can be the earliest clinical manifestation of pancreatic cancer $(31,32,43)$, but it may be difficult to distinguish between pancreatic inflammation caused by AP and pancreatic tumors (44), even if using CECT and MRI. At this time, it may be mistaken for pancreatitis caused by gallstones and alcohol, rather than pancreatitis caused by the original pancreatic cancer (45).

AP caused by other benign and malignant pancreatic tumors is relatively rare, and their pathogenesis requires more in-depth research, such as NETs and ICTs. NETs are a rare cause of $\mathrm{AP}$, and the location of the primary tumor has nothing to do with the severity of AP. Case reports on pancreatitis secondary to pancreatic NETs are rare (46). The etiology of pancreatitis in pancreatic NETs is still unclear. It is most likely due to pancreatic infarction, presumably secondary to the local blood vessels infiltrated by the tumor, and thrombosis in the splenic vein would support this theory (47). ICT is also a rare cause of AP, which may be caused by the occurrence of pancreatic duct obstruction (48). Pancreatic cancer and other primary or metastatic pancreatic tumors can induce episodes of AP, but the exact underlying mechanism, its erratic development, and the extent of the secondary inflammatory response produced by the tumor are likely to be different, and still remains controversial $(42,49,50)$.

Another manifestation of PTP, that is, pancreatitis was not found before pancreatic tumor surgery, and severe pancreatic inflammation was found during the operation. It is a type worthy of further discussion, as severe inflammation not only affects the surgical process, but also affects postoperative recovery. For example, we report a 55-year-old female patient who presented to the First Affiliated Hospital of China Medical University with "abdominal pain and bloating for 1 month" as the main complaint, and the abdominal enhanced CT showed that the pancreatic duct is dilated, a low-density shadow with a diameter of $1.6 \mathrm{~cm}$ is seen at the head of the pancreas near the ampulla of Vater, and after enhancement, the edge is irregularly enhanced and a weakly enhanced area is seen inside. After doing the preoperative examination and preoperative evaluation, a pancreaticoduodenectomy was performed, and inflammation was seen during the operation (Figure 1). The postoperative histopathological diagnosis was well-differentiated ductal adenocarcinoma. The patient's pancreatic inflammation was severe, representing a typical PTP case.

When pancreatitis recurs, PTP should be considered. A previous study showed that most patients have AP recurrence before the diagnosis of IPMN (35). This may be due to the fact that changes of the pancreatic duct are subtle and minimal in the early stage of IPMN, and PTP can mistakenly be regarded as idiopathic pancreatitis. The relationship between the tumor and AP is worth further study. Is AP caused by tumors, or does pancreatitis, which is also a risk factor for pancreatic tumors, cause pancreatic tumors? Will PTP accelerate the progression of pancreatic tumors? There is less literature on these aspects. A 2018 study showed that a history of AP may accelerate the development of pancreatic cancer (51). A cohort study involving 41,669 patients with AP showed that $\mathrm{AP}$ should be considered as a risk factor for the subsequent development of pancreatic cancer, and the absolute risk of pancreatic cancer in patients with $\mathrm{AP}$ at 2 and 5 years was $0.70 \%$ and $0.87 \%$, respectively (52). A 2017 cohort study showed that the increase in the number of recurrences of $\mathrm{AP}$ is an independent risk factor for pancreatic cancer. If 

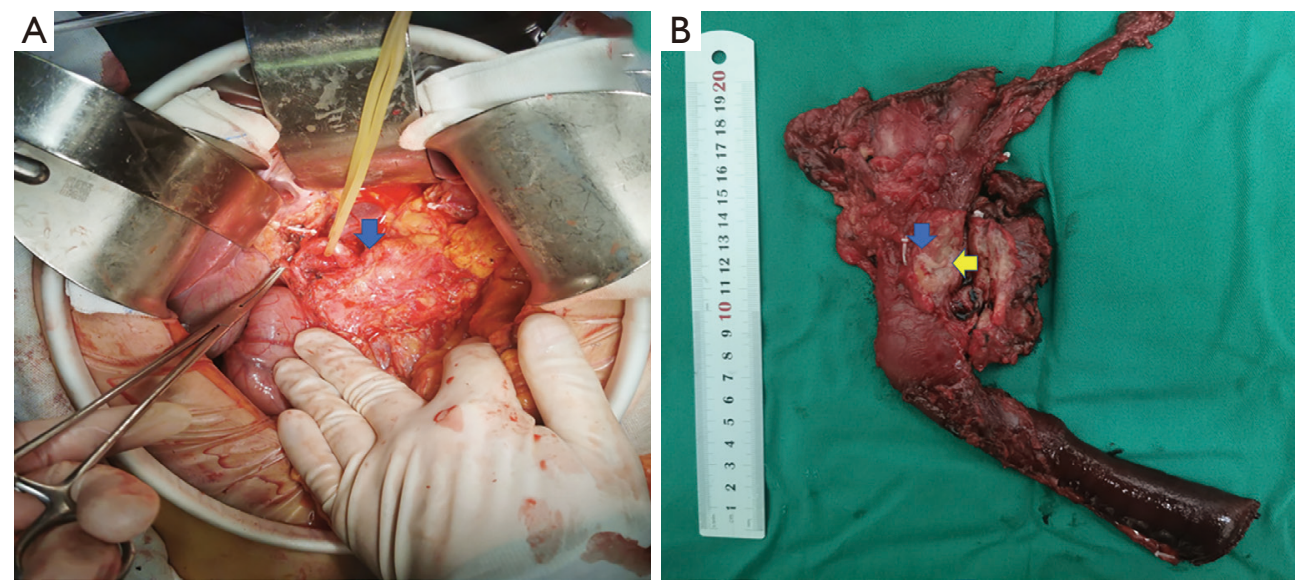

Figure 1 Intraoperative inflammation in a typical PTP patient. (A) Pancreatic head tumor-associated pancreatitis; the blue arrow shows AP. (B) The blue arrow shows AP; yellow arrows are visible for pancreatic head cancer. PTP, pancreatic tumor-associated pancreatitis; AP, acute pancreatitis.

the clinical manifestations are consistent with AP, these findings mean that the diagnosis of pre-existing pancreatic cancer is delayed (53). Although pancreatitis increases the risk of pancreatic cancer, does pancreatitis inevitably lead to pancreatic tumors? Is pancreatic tumors an inevitable result of pancreatitis? We believe that it is not yet possible to prove that there is an inevitable causal relationship between them. This is an imaginative problem that requires enough research to confirm.

\section{Conclusions}

$\mathrm{AP}$ is a common and potentially life-threatening inflammatory disease of the pancreas. PTP is a new classification method for pancreatitis, which has its own unique classification advantages. It can be concluded that the pathophysiological mechanism of PTP may be different from pancreatic tumors and may differ among individuals. Its triggering mechanisms, mainly pancreatic duct obstruction, extravasation, and the activation of pancreatic enzymes, are not completely understood, but there is no doubt that the relationship between pancreatitis and pancreatic tumors should be recognized. PDAC or other primary or metastatic pancreatic tumors can cause single or recurrent AP. PDAC and IPMN are the two pancreatic tumors most related to PTP. In addition to the treatment of general pancreatitis, PTP also requires surgical treatment. The surgical treatment of PTP requires attention: (I) mild to moderate pancreatitis with pancreatic malignancies, even if the operation is difficult, must be operated, and with benign tumors, pancreatitis can be treated first; (II) for severe pancreatitis combined with pancreatic tumor, that is, severe PTP, regardless of whether the tumor is benign or malignant, pancreatitis must be treated first; (III) in our clinical work and case summary, we found that most of the PTP cases have mild to moderate pancreatitis, and there are fewer patients with severe pancreatitis. This may be related to the long process of pancreatitis during the development of pancreatic tumors. The PTP patients who are manifested as AP may be early surgical resection due to early detection of pancreatic tumors, thereby improving the survival rate of patients. PTP is proposed in the hope that clinicians can gain some experience and inspiration, so that patients can obtain better treatment results. The question of the most optimal treatment method for PTP still remains. The priority of pancreatitis and pancreatic tumor treatment is worth discussing. The optimal timing of surgery also requires more research for clarification. Finally, future research should design the best diagnostic and treatment algorithm for PTP.

\section{Acknowledgments}

Funding: Liaoning Provincial Department of Education Science Research Project (No. L2014299) and National Natural Science Foundation of China (No. 81572360).

\section{Footnote}

Reporting Checklist: The authors have completed the 
Narrative Review reporting checklist. Available at https:// dx.doi.org/10.21037/gs-21-400

Conflicts of Interest: All authors have completed the ICMJE uniform disclosure form (available at https://dx.doi. org/10.21037/gs-21-400). All authors report funding from Liaoning Provincial Department of Education Science Research Project (No. L2014299) and National Natural Science Foundation of China (No. 81572360). The authors have no other conflicts of interest to declare.

Ethical Statement: The authors are accountable for all aspects of the work in ensuring that questions related to the accuracy or integrity of any part of the work are appropriately investigated and resolved.

Open Access Statement: This is an Open Access article distributed in accordance with the Creative Commons Attribution-NonCommercial-NoDerivs 4.0 International License (CC BY-NC-ND 4.0), which permits the noncommercial replication and distribution of the article with the strict proviso that no changes or edits are made and the original work is properly cited (including links to both the formal publication through the relevant DOI and the license). See: https://creativecommons.org/licenses/by-nc-nd/4.0/.

\section{References}

1. Xiao AY, Tan ML, Wu LM, et al. Global incidence and mortality of pancreatic diseases: a systematic review, metaanalysis, and meta-regression of population-based cohort studies. Lancet Gastroenterol Hepatol 2016;1:45-55.

2. Forsmark CE, Vege SS, Wilcox CM. Acute pancreatitis. N Engl J Med 2016;375:1972-81.

3. Petrov MS, Yadav D. Global epidemiology and holistic prevention of pancreatitis. Nat Rev Gastroenterol Hepatol 2019;16:175-84.

4. Nesvaderani M, Eslick GD, Vagg D, et al. Epidemiology, aetiology and outcomes of acute pancreatitis: a retrospective cohort study. Int J Surg 2015;23:68-74.

5. Roberts SE, Morrison-Rees S, John A, et al. The incidence and aetiology of acute pancreatitis across Europe. Pancreatology 2017;17:155-65.

6. van Dijk SM, Hallensleben NDL, van Santvoort HC, et al. Acute pancreatitis: recent advances through randomised trials. Gut 2017;66:2024-32.

7. Boxhoorn L, Voermans RP, Bouwense SA, et al. Acute pancreatitis. Lancet 2020;396:726-34.
8. Dumonceau JM, Andriulli A, Deviere J, et al. European Society of Gastrointestinal Endoscopy (ESGE) Guideline: prophylaxis of post-ERCP pancreatitis. Endoscopy 2010;42:503-15.

9. Yadav D, Lowenfels AB. The epidemiology of pancreatitis and pancreatic cancer. Gastroenterology 2013;144:1252-61.

10. Gukovskaya AS, Pandol SJ, Gukovsky I. New insights into the pathways initiating and driving pancreatitis. Curr Opin Gastroenterol 2016;32:429-35.

11. Gerasimenko JV, Gryshchenko O, Ferdek PE, et al. Ca2+ release-activated $\mathrm{Ca} 2+$ channel blockade as a potential tool in antipancreatitis therapy. Proc Natl Acad Sci U S A 2013;110:13186-91.

12. Wen L, Voronina S, Javed MA, et al. Inhibitors of ORAI1 prevent cytosolic calcium-associated injury of human pancreatic acinar cells and acute pancreatitis in 3 mouse models. Gastroenterology 2015;149:481-92.e7.

13. Aghdassi AA, John DS, Sendler M, et al. Cathepsin D regulates cathepsin $\mathrm{B}$ activation and disease severity predominantly in inflammatory cells during experimental pancreatitis. J Biol Chem 2018;293:1018-29.

14. Sendler M, Weiss FU, Golchert J, et al. Cathepsin B-mediated activation of trypsinogen in endocytosing macrophages increases severity of pancreatitis in mice. Gastroenterology 2018;154:704-18.e10.

15. Xu LL, Zhao B, Sun SL, et al. High-dose vitamin C alleviates pancreatic injury via the NRF2/NQO1/HO-1 pathway in a rat model of severe acute pancreatitis. Ann Transl Med 2020;8:852.

16. Javed MA, Wen L, Awais M, et al. TRO40303 Ameliorates alcohol-induced pancreatitis through reduction of fatty acid ethyl ester-induced mitochondrial injury and necrotic cell death. Pancreas 2018;47:18-24.

17. Biczo G, Vegh ET, Shalbueva N, et al. Mitochondrial dysfunction, through impaired autophagy, leads to endoplasmic reticulum stress, deregulated lipid metabolism, and pancreatitis in animal models. Gastroenterology 2018;154:689-703.

18. Zheng Z, Ding YX, Qu YX, et al. A narrative review of acute pancreatitis and its diagnosis, pathogenetic mechanism, and management. Ann Transl Med 2021;9:69.

19. Wu JS, Li WM, Chen YN, et al. Endoplasmic reticulum stress is activated in acute pancreatitis. J Dig Dis 2016;17:295-303.

20. Jakkampudi A, Jangala R, Reddy BR, et al. NF- B in acute pancreatitis: Mechanisms and therapeutic potential. Pancreatology 2016;16:477-88. 
21. Merza M, Hartman H, Rahman M, et al. Neutrophil extracellular traps induce trypsin activation, inflammation, and tissue damage in mice with severe acute pancreatitis. Gastroenterology 2015;149:1920-31.e8.

22. Sun H, Zuo HD, Lin Q, et al. MR imaging for acute pancreatitis: the current status of clinical applications. Ann Transl Med 2019;7:269.

23. Lee PJ, Papachristou GI. New insights into acute pancreatitis. Nat Rev Gastroenterol Hepatol 2019;16:479-96.

24. Al-Bahrani AZ, Ammori BJ. Clinical laboratory assessment of acute pancreatitis. Clin Chim Acta 2005;362:26-48.

25. Tenner S, Baillie J, DeWitt J, et al. American College of Gastroenterology guideline: management of acute pancreatitis. Am J Gastroenterol 2013;108:1400-15; 1416.

26. Crockett SD, Wani S, Gardner TB, et al. American Gastroenterological Association Institute Guideline on initial management of acute pancreatitis. Gastroenterology 2018;154:1096-101.

27. Isaji S, Takada T, Mayumi T, et al. Revised Japanese guidelines for the management of acute pancreatitis 2015: revised concepts and updated points. J Hepatobiliary Pancreat Sci 2015;22:433-45.

28. Working Group IAP/APA Acute Pancreatitis Guidelines. IAP/APA evidence-based guidelines for the management of acute pancreatitis. Pancreatology 2013;13:e1-15.

29. Balthazar EJ. Pancreatitis associated with pancreatic carcinoma. Preoperative diagnosis: role of CT imaging in detection and evaluation. Pancreatology 2005;5:330-44.

30. Mofidi R, Duff MD, Wigmore SJ, et al. Association between early systemic inflammatory response, severity of multiorgan dysfunction and death in acute pancreatitis. $\mathrm{Br}$ J Surg 2006;93:738-44.

31. Lin A, Feller ER. Pancreatic carcinoma as a cause of unexplained pancreatitis: report of ten cases. Ann Intern Med 1990;113:166-7.

32. Zhao X, Lang R, Zhang Z, et al. Exploring and validating the clinical risk factors for pancreatic cancer in chronic pancreatitis patients using electronic medical records datasets: three cohorts comprising 2,960 patients. Transl Cancer Res 2020;9:629-38.

33. Tanaka M, Kobayashi K, Mizumoto K, et al. Clinical aspects of intraductal papillary mucinous neoplasm of the pancreas. J Gastroenterol 2005;40:669-75.

34. Venkatesh PG, Navaneethan U, Vege SS. Intraductal papillary mucinous neoplasm and acute pancreatitis. J Clin Gastroenterol 2011;45:755-8.

35. Pelletier AL, Hammel P, Rebours V, et al. Acute pancreatitis in patients operated on for intraductal papillary mucinous neoplasms of the pancreas: frequency, severity, and clinicopathologic correlations. Pancreas 2010;39:658-61.

36. Zamora C, Sahel J, Cantu DG, et al. Intraductal papillary or mucinous tumors (IPMT) of the pancreas: report of a case series and review of the literature. Am J Gastroenterol 2001;96:1441-7.

37. Kida A, Shirota Y, Suda T, et al. A case of macrocystic-type serous cystic neoplasm with repeated pancreatitis within a short period of time. Clin J Gastroenterol 2017;10:558-63.

38. Shioyama E, Mitoro A, Ogawa $\mathrm{H}$, et al. A pancreatic mucinous cystic neoplasm undergoing intriguing morphological changes over time and associated with recurrent pancreatitis: a case report. Medicine (Baltimore) 2019;98:e16435.

39. Chikuie E, Fukuda S, Tazawa H, et al. A solid pseudopapillary neoplasm of the pancreas in a man presenting with acute pancreatitis: a case report. Int J Surg Case Rep 2017;31:114-8.

40. Ozturk Y, Soylu OB, Gurcu B, et al. Solid pseudopapillary tumor of the pancreas as a cause of recurrent pancreatitis. Acta Gastroenterol Belg 2008;71:390-2.

41. Munigala S, Kanwal F, Xian H, et al. Increased risk of pancreatic adenocarcinoma after acute pancreatitis. Clin Gastroenterol Hepatol 2014;12:1143-50.e1.

42. Gambill EE. Pancreatitis associated with pancreatic carcinoma: a study of 26 cases. Mayo Clin Proc 1971;46:174-7.

43. Li S, Tian B. Acute pancreatitis in patients with pancreatic cancer: timing of surgery and survival duration. Medicine (Baltimore) 2017;96:e5908.

44. Frampas E, Morla O, Regenet $\mathrm{N}$, et al. A solid pancreatic mass: tumour or inflammation? Diagn Interv Imaging 2013;94:741-55.

45. Lee ES, Lee JM. Imaging diagnosis of pancreatic cancer: a state-of-the-art review. World J Gastroenterol 2014;20:7864-77.

46. Tejedor Bravo M, Justo LM, Lasala JP, et al. Acute pancreatitis secondary to neuroendocrine pancreatic tumors: report of 3 cases and literature review. Pancreas 2012;41:485-9. Erratum in: Pancreas 2012;41:825.

47. Jamieson A, Connell JM. Neuroendocrine pancreatic cancer: an unusual case of pancreatitis. Scott Med J 2000;45:55-6.

48. Ramsay D, Gibson P, Edmunds S, et al. Pancreatic islet cell tumours presenting as recurrent acute pancreatitis: imaging features in three cases. Australas Radiol 
2001;45:520-3.

49. Levine E. Carcinoma of the pancreas presenting as acute pancreatitis: CT diagnosis. Gastrointest Radiol 1981;6:29-33.

50. van Gulik TM, Moojen TM, van Geenen R, et al. Differential diagnosis of focal pancreatitis and pancreatic cancer. Ann Oncol 1999;10 Suppl 4:85-8.

51. Phillips AE, Shah N, Borhani AA, et al. Prior history of pancreatitis accelerates the development of pancreatic adenocarcinoma. Pancreas 2018;47:1262-6.

Cite this article as: Gou A, Liu Z, Xiao Z, Li G, Xu Y, Song S, Guo K, Ma G. A narrative review of a type of pancreatitis worthy of attention: acute pancreatitis associated with pancreatic tumors-current problems and future thinking. Gland Surg 2021;10(7):2304-2312. doi: 10.21037/gs-21-400
52. Kirkegård J, Cronin-Fenton D, Heide-Jørgensen U, et al. Acute pancreatitis and pancreatic cancer risk: a nationwide matched-cohort study in Denmark. Gastroenterology 2018;154:1729-36.

53. Sadr-Azodi O, Oskarsson V, Discacciati A, et al. Pancreatic cancer following acute pancreatitis: a population-based matched cohort study. Am J Gastroenterol 2018;113:1711-9.

(English Language Editor: C. Betlazar-Maseh) 